\title{
Healthcare Workers Infection Rate in the Era of Coronavirus Disease 2019 - in Tertiary Teaching Hospital
}

\author{
Mona Mohiedden ${ }^{1}$ D, Aml M. Said ${ }^{1}$, Ahmed M. Ali², Mohammed M. Abdel Razik ${ }^{3}$, Maha Ali Gad ${ }^{1}$ (D) \\ ${ }^{1}$ Department of Clinical and Chemical Pathology, Faculty of Medicine, Cairo University, Cairo, Egypt; ${ }^{2}$ Department of Obstetrics \\ and Gynecology, Faculty of Medicine, Cairo University, Cairo, Egypt; ${ }^{3}$ Department of Public Health and Community Medicine, \\ Faculty of Medicine, Cairo University, Cairo, Egypt
}

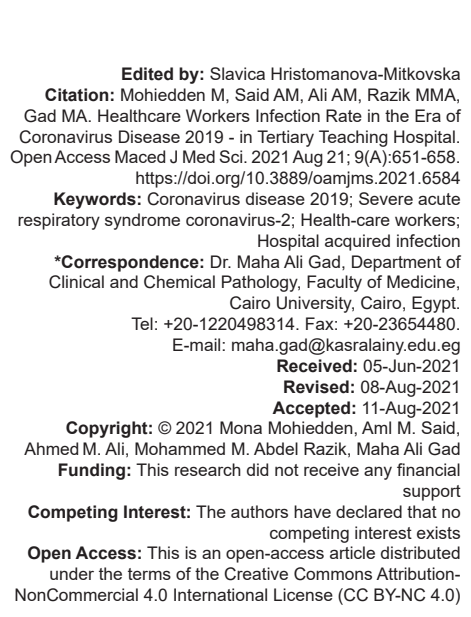

\begin{abstract}
BACKGROUND: Healthcare workers (HCWs) are at the frontline defense against coronavirus disease 2019 (COVID-19) pandemic.

AIM: The study aimed to describe the characteristics and appraise potential risk factors of COVID-19 transmission among HCWs who tested positive for severe acute respiratory syndrome coronavirus-2 (SARS-CoV-2) in one of Cairo University Hospitals.

METHOD: Cross-sectional descriptive analysis of confirmed polymerase chain reaction (PCR) positive versus negative cases for COVID-19.

RESULTS: Through March-June 2020, (145/846; 17\%) suspected HCWs were tested for COVID-19 by PCR; out of them $(70 / 145 ; 48.3 \%)$ were confirmed as positive, these positive cases represented $(70 / 846 ; 8.3 \%)$ of all HCWs of the hospital. About $33 \%$ of confirmed COVID-19 positive HCWs acquired the infection from the healthcare while only $(13 / 70 ; 19 \%)$ from community settings, and no clear exposure data were identified in $(34 / 70 ; 48 \%)$ of cases. Most of symptomatic cases showed a positive PCR test for SARS-CoV-2 versus asymptomatic cases, $p<0.001$. There was no statistical significance regarding gender, age, presence of comorbidity, workload or the type of acquisition

CONCLUSION: HCWs are at an increased risk of COVID-19 infection at the workplace. Strict implementation of infection control measures is of crucial role in preventing transmission of COVID-19 infection in health-care settings.
\end{abstract}

\section{Introduction}

According to the World Health Organization (WHO), the outbreak of coronavirus disease 2019 (COVID-19) has become a pandemic, which at the time of writing had affected a huge number of people and caused many deaths worldwide [1], [2].

During pandemic of COVID-19, one of the most important sectors that are affected is health-care workers ( $\mathrm{HCWs}$ ) as they are always in the frontline facing many infectious diseases; they are at increased risk of being exposed to severe acute respiratory syndrome coronavirus-2 (SARS-CoV-2) and can potentially have a role in hospital transmission [3].

Risk factors for transmission of infection among HCWs mostly include overcrowding, lack of standard isolation room facilities, improper implementation of infection control measures specially in rest rooms in break periods, and improper environmental hygiene protocols [4]. During the periods of outbreak of COVID-19 or other infectious diseases, implementation of strict infection prevention and control (IPC) measures is playing crucial part in the inhibition of spread of these viruses among HCWs in health-care settings, especially regarding personal protection.

The impending shortage of personal protective equipment (PPE) and the proposed changes in its use in response to increase demands is also possible risks to HCWs at the era of the pandemic [5], [6], [7].

A common misunderstanding among many end users is that they are protected from potentially infectious materials when they wear any type of fluidresistant protective equipment [8]. It is mandatory for every healthcare facility, especially with concordant limitation resources mainly PPEs during pandemic, the noticed malpractice and inappropriate attitude due to physical and psychological stress [9].

We aimed to assess infection rate of COVID19 among HCWs since March 2020, with the beginning of COVID-19 first wave in Egypt and for the following 5 months, at Obstetrics and Gynaecology Hospital one of the Teaching University Hospitals in addition to appraisal of potential risk factors of its transmission. 


\section{Subjects and Methods}

\section{Study design}

A cross-sectional descriptive study was conducted from March-June 2020 at one of the tertiary care referral center in Egypt which provides health services in all specialties with about 5600 beds. It includes outpatient clinics and emergency departments. One of the important affiliated hospitals is obstetrics and gynecology hospital which serves about 1000 females per month through outpatient clinics or emergency sector.

We aimed to detect infection rate of COVID19 among HCWs in Obstetrics and Gynaecology hospital. The study described the characteristics HCWs who tested positive for COVID-19 in our hospital with analysis of risk factors, and the exposure details.

Confirmed reverse transcription-polymerase chain reaction (RT-PCR) positive COVID-19 HCWs are isolated in accordance according to their clinical condition at home or hospital. They are allowed to return back to work after 14 days from being confirmed as positive for COVID-19 and they should be symptoms- free with negative RT-PCR for SARS-CoV-2 according to the WHO recommendations. The RT-PCR negative COVID$19 \mathrm{HCWs}$ are followed by laboratory tests, computerized tomography (CT) chest as required according to their clinical condition, with repetition of NSP swab after $48 \mathrm{~h}$ to reduce possibility of false negative results.

\section{Study outcome}

\section{Primary outcome}

Determine infection rate of COVID-19 among HCWs in one of Cairo University Hospitals (CUHs) during the COVID-19 wave and make in-depth analysis of sources and modes of transmission of SARS-CoV-2 among HCWs.

\section{Secondary outcome}

Characterize both symptomatic and asymptomatic HCWs after being confirmed positive by real-time RT-PCR for SARS-CoV-2.

\section{Study population}

Our hospital is specialized in gynecology and obstetrics accordingly potentially infected COVID-19 cases would be admitted at any time for specialized gynecological and obstetric medical care. Infection control committee with support of the hospital administration has settled strict policy and precautions and the workflow has been also rearranged to protect the frontline obstetricians, $\mathrm{HCWs}$ and patients in the era of COVID-19 pandemic. Most services were postponed except emergencies and non-deferrable cases. All patients present to the hospital during the pandemic, should be screened for symptoms or risk factors for COVID-19 following precise triaging criteria, and any suspected or confirmed case which does not need special care should be referred to the assigned isolation hospitals for COVID-19 cases. Any critical case which need specialized gynecological or obstetric management and meet the criteria of suspected or confirmed COVID-19 should be admitted to the hospital in designed isolation rooms for COVID-19 cases for primary assessment and management by dedicated well trained team only deals with these cases, then referred to assigned isolation hospitals after the cases are stable.

There are $846 \mathrm{HCWs}$ working in the hospital including; 116 clinicians,141 house officers, 22 pharmacists, 370 nurses, among them about 120 newly trained nurse candidates, 81 administrative employees, and 116 paramedics were involved in the cross sectional study.

The protocol of our hospital is to monitor closely all HCWs, including staff working in the triage screening areas and the dedicated team for caring of COVID- 19 cases by infection control professionals of the hospital for any signs or symptoms of SARS-CoV-2 infection. Symptomatic and/or suspected personnel according to specific criteria are segregated from work and they are eligible to nasopharyngeal swab (NSP) which is tested by RT-PCR for SARS-CoV-2. Furthermore, HCWs who give history of contact with confirmed COVID-19 case either in the hospital or outside are also eligible to NSP swabbing irrespective of being symptomatic or not.

\section{Questionnaire design for $\mathrm{HCWs}$}

Epidemiological data of $\mathrm{HCWs}$ were collected through the previously mentioned structured questionnaire (attached) which was prepared by the team of the study as part of their study routine work and analysis of the current pandemic. The following variables were included: Risk factors, comorbidity, transmission models of COVID-19 among HCWs, medical history, exposure to suspected or confirmed COVID-19 cases, and symptoms compatible with COVID-19.

\section{Sampling process}

SARS-CoV-2 laboratory tests are performed following recommendations of the WHO. NSP swabs are obtained by nurses using standard technique of wiping a flocculated swab across the posterior oropharynx from 1 tonsillar area to the other with strict infection control precautions. Swabs are immediately immersed in transport medium, transferred to the hospital molecular microbiology laboratory in portable 
ice-box. VIASURE SARS-CoV-2 Real-Time PCR detection kit (CerTest, BIOTEC) is used for detection of COVID-19 in respiratory samples. It is done in one step real-time RT format where the reverse transcription and the subsequent amplification of target sequence ORF1 $\mathrm{ab}$ and $\mathrm{N}$ genes occur in the same reaction well using specific primers and a fluorescent-labeled probe.

\section{Statistical analysis}

Data entry and analysis was performed through the Statistical Package of the Social Sciences Software program, version 25 (IBM SPSS Statistics for Windows, Version 25.0. Armonk, NY: IBM Corp.). Data were presented using mean and standard deviation for quantitative variables and frequency and percentage for qualitative ones. Comparison between groups for qualitative variables was performed using Chi-square or Fisher's exact tests while for quantitative variables the comparison was conducted using independent sample t-test or Mann-Whitney test. $\mathrm{P}$ values less than or equal to 0.05 were considered statistically significant.

\section{Results}

Out of the $846 \mathrm{HCWs}, 145 \mathrm{HCWs}$ were suspected for COVID-19 infection (17\%). They were distributed as $(46 / 145,31.7 \%)$ among physicians, (78/145, 53.8\%) among nurses, (16/145, 11\%) among patient transporters/cleaners, and $(3 / 145,2.1 \%)$ among administrative employees. Of which $27 \%$ of suspected HCWs had comorbidity as respiratory diseases, diabetes, and cardiac diseases $(10.3 \%, 7.6 \%$, and $7.6 \%$, respectively) in their medical history.

About $61 \%$ of our study group worked at hot areas which include emergency room, operation rooms and ICU. About $63 \%$ of suspected HCWs were frontline.

Most of suspected tested HCWs were symptomatic $(75.9 \%)$. Out of them, $78(54 \%)$ persons reported exposure to suspected or confirmed COVID19 cases either in the hospital or outside in the previous 2 weeks. About (56/78, 72\%) of exposure were reported inside the hospital.

Contact with infected colleagues during break or at residency rooms, examine suspected infected patients or sampling; these events represented $(34.6 \%$, $15.4 \%$, and $10.3 \%$, respectively) of exposure incidents in the hospital. Only $28 \%$ reported contact with friend or infected family members outside hospital.

Regarding training of HCWs during that critical time, we mainly focused on hand hygiene, donning and doffing of PPE. 117 out of 145 suspected HCWs in this study $(80.6 \%)$ received infection control training.
Among studied HCWs; the confirmed positive for COVID-19 by RT-PCR was (70/846, $8.3 \%$ ) which represented $(70 / 145,48.3 \%)$ of our studied suspected HCWs. 46/70, 66\% worked at frontline and 43/70, $61.4 \%$ of them worked at hot areas. The most affected group was the nurses followed by doctors and workers (55.7\%, 28.6\%, and $15.7 \%$, respectively). 51\%, 36/70 of confirmed positive HCWs to COVID-19 reported exposure to suspected and/or confirmed COVID-19 individuals; most of these exposure incidents occurred at hospital settings (23/36, 64\%). Table 1 shows comparison between group of positive and negative RT-PCR for SARS-CoV-2.

The table reflects the importance of IPC training as a significant difference between PCR negative versus positive COVID-19 cases among suspected HCWs. Suspicion was more common among frontline individuals, and personnel who were working in the hot areas and contact cases especially in the hospital; but without a significant difference between positive and negative groups.

Out of 145 suspected HCWs, the duration between exposure and developing symptoms was 2-14 days. Ninety percent (60/70) of cases confirmed as COVID-19 positive showed symptoms within $5.4 \pm 2.3$ days of exposure. Fever and cough were the most common symptoms among suspected workers ( $44.8 \%$ and $41.4 \%$, respectively). Fever was the most commonly detected symptom among HCWs confirmed as positive for COVID-19 (49/70; 70\%) Table 2.

Table 2: Clinical data of HCWs with positive versus negative PCR for SARS-CoV-2

Table 1: Demographic, work criteria, and exposure information of the suspected HCWs included in the study

\begin{tabular}{|c|c|c|c|}
\hline & \multicolumn{2}{|l|}{ COVID-19 PCR } & \multirow[t]{2}{*}{$\mathrm{p}$-value } \\
\hline & $\begin{array}{l}\text { Positive }(\mathrm{n}=70) \\
\mathrm{n}(\%)\end{array}$ & $\begin{array}{l}\text { Negative }(\mathrm{n}=75) \\
\mathrm{n}(\%)\end{array}$ & \\
\hline \multicolumn{3}{|l|}{ Gender } & 0.675 \\
\hline Male & $16(22.9)$ & $15(20)$ & \\
\hline Female & 54 (77.1) & $60(80)$ & \\
\hline Age & $37 \pm 9.7$ & $35.1 \pm 10.8$ & 0.250 \\
\hline$<40$ years & $38(54.3)$ & $49(65.3)$ & 0.175 \\
\hline 40 years or more & $32(45.7)$ & $26(34.7)$ & \\
\hline \multicolumn{3}{|l|}{ Experience (years) } & 0.037 \\
\hline$<5$ & $20(28.6)$ & $34(45.3)$ & \\
\hline$>5$ & $50(71.4)$ & $41(54.7)$ & \\
\hline \multicolumn{3}{|l|}{ Work location } & 0.584 \\
\hline Frontline & $46(65.7)$ & $46(61.3)$ & \\
\hline Non-Frontline & $24(34.3)$ & $29(38.7)$ & \\
\hline \multicolumn{3}{|l|}{ Workload* } & 0.870 \\
\hline High & $28(40)$ & $31(41.3)$ & \\
\hline Low & $42(60)$ & $44(58.7)$ & \\
\hline \multicolumn{3}{|l|}{ Job title } & 0.719 \\
\hline Nurses & $39(55.7)$ & $39(52)$ & \\
\hline Doctors & $20(28.6)$ & $26(34.7)$ & \\
\hline Workers & $11(15.7)$ & $10(13.3)$ & \\
\hline \multicolumn{3}{|l|}{ Site of working } & 0.860 \\
\hline Hot areas ${ }^{\star \star}$ & $43(61.4)$ & $45(60)$ & \\
\hline Cold areas & 27 (38.6) & $30(40)$ & \\
\hline Exposure type & $36(51.4)$ & $42(56)$ & 0.581 \\
\hline Hospital exposure & $23(63.9)$ & $33(78.6)$ & 0.151 \\
\hline Community exposure & $13(36.1)$ & $9(21.4)$ & \\
\hline Receiving IC training & 51 (72.9) & $66(88)$ & 0.021 \\
\hline \multicolumn{4}{|l|}{ Type of training } \\
\hline Course & $4(7.8)$ & $15(22.7)$ & 0.036 \\
\hline Lecture & $22(43.1)$ & $31(47)$ & \\
\hline On job training & $25(49)$ & $20(30.3)$ & \\
\hline
\end{tabular}




\begin{tabular}{|c|c|c|c|}
\hline & \multicolumn{2}{|l|}{ COVID-19 PCR } & \multirow[t]{2}{*}{$p$-value } \\
\hline & $\begin{array}{l}\text { Positive }(\mathrm{n}=70) \\
\mathrm{n}(\%)\end{array}$ & $\begin{array}{l}\text { Negative }(n=75) \\
n(\%)\end{array}$ & \\
\hline Comorbidity & $22(31.4)$ & $17(22.7)$ & 0.234 \\
\hline Respiratory diseases & $8(11.4)$ & $7(9.3)$ & 0.678 \\
\hline DM & $6(8.6)$ & $5(6.7)$ & 0.665 \\
\hline Cardiac diseases & $5(7.1)$ & $6(8)$ & 0.846 \\
\hline Case presentation & & & 0.000 \\
\hline Symptomatic & $63(90)$ & $47(62.7)$ & \\
\hline Asymptomatic & $7(10)$ & $28(37.3)$ & \\
\hline Timing of symptoms (days) & $5.4 \pm 2.3$ & $5.2 \pm 3$ & 0.637 \\
\hline \multicolumn{4}{|l|}{ Symptoms } \\
\hline Fever & $49(70)$ & $16(21.3)$ & 0.000 \\
\hline Sore throat & $23(32.9)$ & $24(32)$ & 0.912 \\
\hline Cough & $39(55.7)$ & $21(28)$ & 0.001 \\
\hline Dyspnea & $30(42.9)$ & $13(17.3)$ & 0.001 \\
\hline Myalgia and bony ache & 30 (42.9) & $16(21.3)$ & 0.005 \\
\hline Diarrhea & $19(27.1)$ & $17(22.7)$ & 0.533 \\
\hline Anosmia & $9(12.9)$ & $2(2.7)$ & 0.021 \\
\hline Headache & $7(10)$ & $6(8)$ & 0.674 \\
\hline Pneumonia & $1(1.4)$ & $1(1.3)$ & 1.000 \\
\hline
\end{tabular}

The table shows that comorbidity was more common among PCR positive HCWs for COVID-19, but without statistically significant value. Most of symptomatic cases showed a positive PCR test for SARS-CoV-2 versus asymptomatic cases with statistically significant value. Most of the infected HCWs had satisfactory outcome as $73 \%$ of studied cases were home isolated while only $1.4 \%$ required ICU admission.

\section{Discussion}

Egypt has been hit by this pandemic with the first confirmed case officially announced on 14 February 2020 [10]. The number of cases increased to reach 26,384 cases by June and exceed 80000 cases by July [11], [12]. HCWs have been significantly affected by the pandemic worldwide, as well as in Egypt [13].

In our study, 145 of employees at Obstetrics and Gynaecology hospital were suspected cases of COVID-19. The positive RT-PCR COVID-19 cases represented $8.3 \%$ of all hospital employees and $48.3 \%$ of the suspected HCWs. The rate of infection was high because NSP swab was restricted for high risk HCWs and not for screening of all HCWs.

On the other hand, Kassem et al. declared that $13.5 \%$ of screened HCWs were confirmed positive but the aim of their study was point prevalence surveillance with small sample size [14].

A study in a German university hospital, 52 out of 957 employees (5.4\%) tested positive for COVID-19 by PCR [15]. In a Dutch study, $14 \% \mathrm{HCW}$ were symptomatic suspected cases and $6 \%$ of them tested positive for SARS-CoV-2 through nasal swab [16]. This variety could be explained by different study designs of $\mathrm{HCWs}$ regarding their work place, being frontline or not, timing of the samples collection, and duration of the study.

In our study, $65 \%$ of the lab confirmed cases were frontline HCWs, $61 \%$ out of them worked at hot area which agrees with our knowledge about this virus and its mode of transmission [17], [18]. Unexpectedly, workload was not one of the risk factors to get COVID19 infection in this study which disagrees with the study of Wang et al., who reported that high workload with long-time exposure to large numbers of infected patients directly increased the risk of infection for HCWs [7]. This could be explained by following strict adherence to infection control measures all the time during work hours while protective measures in HCWs' hostels were not sufficient, in addition to lack of keeping reasonable distance between each other while talking, eating or praying, moreover the doffing of PPEs without caution, non-compliance with hand hygiene and environmental disinfection all these factors may result in exposure to high viral load and increase risk of infection in hostels rather than patient care areas irrespective to work hours.

Among our tested HCWs, neither age nor gender was risk factor for COVID-19 infection and most of the positive cases were among nurse group. This is consistent with Kassem et al. study [14] and could be explained by excess workload difficult comprehending under stress, educational, culture and social status, inadequate nurse to patient ratio, especially at early stage of the pandemic before identification of potential risks of COVID-19 transmission, rigorous implementation and monitoring of infection control measures and before dealing with the problem of PPEs shortage.

A study in India on COVID-19 in HCWs found that, commonly effected age group to be $26-41$ years $(61 \%)$. Understanding the dynamics of SARS-CoV-2 infection in this group is essential to guide formulation of effective infection control measures [3], [19].

In our study, $90 \%$ of COVID-19 PCR positive subjects were symptomatic HCWs. Kassem et al. reported that symptomatic cases represent $73 \%$ of COVID-19 PCR positive results [14].

Furthermore, most positive COVID-19 cases were observed in studies from the United Kingdom and Spain in March 2020, when symptomatic HCWs were tested (18\%) and (38\%) [20], [21]. This is in agreement with high correlation between COVID-19 positivity and the presence of symptoms among HCWs.

These findings may be due to our hospital protocol of testing only suspected HCWs, not mass screening of all HCWs regardless of risk of exposure or suspicion criteria.

Fever, cough, and dyspnea were the most common symptoms among our confirmed COVID19 cases. This is in accordance with many studies in which fever and cough were commonly presented symptoms among COVID-19 cases [16], [22], [23].

Surprisingly COVID- 19 positive cases were significantly higher among $\mathrm{HCWs}$ with more than 5 year experience. This could be explained by work overload on these expertise HCWs and assigning them for management of more severe cases with higher viral 
loads more than younger less expertise HCWs.

Approximately, 54\% suspected COVID-19 individuals reported exposure to suspected or confirmed COVID-19 individuals during work either in the hospital or outside in the past 2 weeks. Regarding confirmed positive COVID-19 cases $33 \%$ were exposed at hospital and $19 \%$ were exposed out of the work areas. This result is in agreement with a study conducted at Germany where 531/957 (55.5\%) suspected employees reported contact with a person tested positive for SARS-CoV-2. Among the employees who tested positive $74.6 \%$ at work, 25.4\% elsewhere [15]. On the other hand, Maskari et al. reported that the most common settings of acquisition of COVID-19 infection among HCWs were community $61.3 \%$, followed by hospital $25.5 \%$ and no source was identified in $13.2 \%$ [20].

Nosocomial transmission should be considered especially among colleagues as many of positive HCWs reported exposure in the hospital environment. However, we cannot exclude role of community as source of COVID-19 infection, cause $36 \%$ of the exposed positive HCWs had history of community contact with suspected or confirmed COVID-19 cases. Same findings were found by Sikkema et al., as they reported that $32 \%$ of $\mathrm{HCW}$ s were in close contact with confirmed case of COVID-19; most of them occurred between colleagues. However, infection of HCWs could be explained by foreign travel, and community contacts [24]. This is great alarm to all population to avoid unnecessary gathering, keeping social distance, compliance with hand hygiene, and strict adherence to proper donning and doffing of appropriate PPEs in hospital and community settings.

Many studies explained the critical measures to reduce nosocomial transmission by respiratory droplets, through strict adherence to hand hygiene and PPE use guidelines by all $\mathrm{HCW}$ s involved in management of confirmed or suspected SARS-CoV-2 infected patients [7], [25]. Sometimes HCWs could not comply with these strategies due to several factors including shortages of medical supplies, inadequate IPC training and cultural factors [5].

Among our suspected HCWs (117/145; 81\%) received IPC training and showed a significant difference between positive and negative tested group. Moreover, IPC interventions such as engineering controls and administrative controls are considered to play a valuable important role in preventing transmission [26].

According to our hospital protocol; the confirmed positive HCWs for COVID-19 are isolated at home for 14 days with close follow-up by assigned team of specialized physicians. Some studies found that HCWs with COVID-19 were more likely to present less severely ill, and less likely to be admitted to the hospital [27], [28]. All recruited HCWs confirmed as COVID-19, in our study, were improved and returned back to work after 2 negative NSP swabs, $48 \mathrm{~h}$ apart [29].

\section{Strengths and limitations}

Our epidemiological data are collected during routine work reflecting real situation and analyzing common mistakes during work for better understanding of the sources of infection and modes of transmission of SARS-CoV-2 among HCWs at one of CUHs, thus guiding infection control professionals to formulate appropriate evidence based IPC measures. However, our study has some limitations; the survey was conducted in one specialized hospital so the results may not be generalizable to other hospital's HCWs. The testing of HCWs was restricted on symptomatic HCWs in the past 14 days and no data were collected from asymptomatic HCWs. In addition, the possibility of false negative result should be considered.

\section{Conclusion}

Mass screening of HCWs during pandemic is a priority. As $48.3 \%$ of our suspected health care workers were positive by PCR test for COVID-19, 51.4\% with history of exposure either community, or at the hospital and $90 \%$ were symptomatic. However, in our limited resource countries at least testing of symptomatic high risk group and those with exposure history is mandatory to avoid addition of HCWs to the burden of COVID-19 infection clusters and transmission among both healthcare and community settings.

Understanding the dynamics of SARS-CoV-2 infection in this population is essential to guide formulation of appropriate and customized IPC measures. Endorse national and local IPC guidelines which recommend levels of PPE based on risk assessment ultimately aiming to provide optimal PPE to all categories of HCWs and preparedness for any coming pandemics.

\section{Acknowledgment}

We would like to thank infection control committee for its heroic role during difficult and hard times including infection control team of Obstetrics and Gynaecology Hospital. Also we appreciate the great commitment and excellent effort of Prof. Mona Salah, Ass Prof. May Sherif and their team at the Molecular Laboratory during pandemic. We cannot express enough our gratitude to all HCWs' efforts and sacrifice during pandemic. 


\section{Authors' Contributions}

All authors were contributed; Dr. Mona and Dr. Maha designed the study, Dr. Maha and Dr. Ahmed provided us with the documents and collected the data. Dr. Mona, Dr. Maha and Dr. Aml carried out the study analysis. Dr. Mostafa prepared the statistical analysis of the study. Dr. Aml and Dr. Ahmed revised and edited the final version of the manuscript. All authors have approved the final article.

\section{Ethical approval}

Ethical approval was obtained from the Medical Ethics Committee- Faculty of Medicine (N-109-2020). History data obtained from the suspected HCWs in addition to SARS-CoV-2 testing results by RT-PCR which were performed as part of the routine infection control policy in response to outbreak situations. All participants were guaranteed anonymity and they have signed written informed consent.

\section{References}

1. Zhang M, Zhou M, Tang F, Wang $\mathrm{Y}$, Nie H, Zhang L, et al. Knowledge, attitude, and practice regarding COVID-19 among healthcare workers in Henan, China. J Hosp Infect. 2020;105(2):183-7. https://doi.org/10.1016/j.jhin.2020.04.012 PMid:32278701

2. World Health Organization. Coronavirus Disease (COVID-19) Pandemic; 2020. Available from: https://www.who.int/ emergencies/diseases/novel-coronavirus-2019. [Last accessed on 2020 Jul 25].

3. Dev N, Kumar V, Sankar J. COVID-19 infection outbreak among health care workers: Perspective from a low-middle income country. Monaldi Arch Chest Dis. 2020;90(3):1474 PMid:32657563

4. Al Abri ZG, Al Zeedi MA, Al Lawati AA. Risk factors associated with COVID-19 infected healthcare workers in Muscat governorate, Oman. J Prim Care Community Health. 2021;12. https://doi.org/10.1177/2150132721995454

PMid:33576288

5. Chersich MF, Gray G, Fairlie L, Eichbaum Q, Mayhew S, Allwood B, et al. COVID-19 in Africa: Care and protection for frontline healthcare workers. Global Health. 2020;16(1):46. https://doi.org/10.1186/s12992-020-00574-3

6. Tan T, Chang C, Chang M. Intra-hospital preventive principles to protect frontline healthcare workers to overcome pandemic COVID-19 in Taiwan. Crit. Care. 2020;24:328. https://doi. org/10.1186/s13054-020-02983-7

7. Wang J, Zhou M, Liu F. Reasons for healthcare workers becoming infected with novel coronavirus disease 2019 (COVID-19) in China. J Hosp Infect. 2020;105(1):100-1. https:// doi.org/10.1016/j.jhin.2020.03.002

PMid:32147406

8. The National Institute for Occupational Safety and Health (NIOSH). Hierarchy of Controls. Center for Disease Control and
Prevention. Available from: https://www.cdc.gov/niosh/topics/ hierarchy/default.html;2020. [Last accessed on $2020 \mathrm{Jul} 13$ ] https://doi.org/10.1037/e371562004-001

9. Nugroho BJ, Soetjahjo B, Heru Nefihancoro U, Ermawan R, Darma Saputra R, Putra GS, et al. Orthopedic department of education center and service provide during coronavirus disease-2019 pandemic: An experience from single-center hospital. Open Access Maced J Med Sci. 2021;9:250-4. https:// doi.org/10.3889/oamjms.2021.6027

10. World Health Organization. Coronavirus Disease 2019 (COVID19)Situation Report-26.Availablefrom: https://www.who.int/docs/ default-source/coronaviruse/situation-reports/20200215- sitrep26-covid-19.pdf?sfvrsn=a4cc6787 2;2020. [Last accessed on 2020 Aug 15].

11. Daily Report on COVID-19 by Egyptian Ministry of Health and Population. Available from: https://www.facebook.com/egypt. mohp/photos/a.123442675873020/177239867159967;2020. [Last accessed on 2020 Aug 15].

12. Daily Report on COVID-19 by Egyptian Ministry of Health and Population. Available from: https://www.facebook.com/egypt. mohp/photos/a.123442675873020/196941851856435;2020 [Last accessed on 2020 Aug 15].

13. Martin C, Montesinos I, Dauby N, Gilles C, Dahma H, Van Den Wijngaert S, et al. Dynamics of SARS-CoV-2 RT-PCR positivity and seroprevalence among high-risk healthcare workers and hospital staff. J Hosp Infect. 2020;106(1):102-6. https://doi. org/10.1016/j.jhin.2020.06.028 PMid:32593608

14. Kassem AM, Talaat H, Shawky S, Fouad R, Amer K, Elnagdy $T$, et al. SARS-CoV-2 infection among healthcare workers of a gastroenterological service in a tertiary care facility. Arab $J$ Gastroenterol. 2020;21(3):151-5. https://doi.org/10.1016/j. ajg.2020.07.005

PMid:32732168

15. Schwierzeck V, Correa-Martinez CL, Schneider KN, Mellmann A Hennies MT, Hafezi W, et al. SARS-CoV-2 in the employees of a large university hospital. Dtsch Arztebl Int. 2020;117(19):344-5. https://doi.org/10.3238/arztebl.2020.0344

PMid:32527380

16. Kluytmans-van den Bergh M, Buiting A, Pas S, Bentvelsen RG van den Bijllaardt W, van Oudheusden AJ, et al. Prevalence and clinical presentation of health care workers with symptoms of coronavirus disease 2019 in 2 Dutch hospitals during an early phase of the pandemic. JAMANetwork Open. 2020;3(5):e209673. https://doi.org/10.1001/jamanetworkopen.2020.9673 PMid:32437576

17. World Health Organization. Coronavirus Disease (COVID-19) Pandemic. Available from: https://www.who.int/ news-room/commentaries/detail/modes-of-transmissionof-virus-causing-covid-19-implications-for-ipc-precautionrecommendations;2020. [Last accessed on 2020 May 03].

18. Li Q, Guan X, Wu P, Wang X, Zhou L, Tong Y, et al. Early transmission dynamics in Wuhan, China, of novel coronavirusinfected pneumonia. N Engl J Med. 2020;382(13):1199-207.

19. Chen $Y$, Tong $X$, Wang J, Huang W, Yin S, Huang R, et al. High SARS-CoV-2 antibody prevalence among healthcare workers exposed to COVID-19 patients. J Infect. 2020;81(3):420-6. https://doi.org/10.1016/j.jinf.2020.05.067 PMid:32504745

20. Keeley AJ, Evans C, Colton H, Ankcorn M, Cope A, State A, et al Roll-out of SARS-CoV-2 testing for healthcare workers at a large NHS foundation trust in the United Kingdom, March 2020. Euro Surveill. 2020;25(14):2000433. https://doi.org/10.2807/15607917.es.2020.25.14.2000433

PMid:32290904

21. Suárez-García I, Martínez de Aramayona López MJ, Sáez 
Vicente A, Lobo Abascal P. SARS-CoV-2 infection among healthcare workers in a hospital in Madrid, Spain. J Hosp Infect. 2020;106(2):357-63. https://doi.org/10.1016/j.jhin.2020.07.020 PMid:32702465

22. Chen N, Zhou M, Dong X, Qu J, Gong F, Han $Y$, et al. Epidemiological and clinical characteristics of 99 cases of 2019 novel coronavirus pneumonia in Wuhan, China: A descriptive study. Lancet. 2020;395(10223):507-13. https://doi.org/10.1016/ s0140-6736(20)30211-7

PMid:32007143

23. Moradali MR, Simbar M, Roozbahani S, Koochaksaraei FY, Hosseinzadeh $\mathrm{P}$, Zareipour $\mathrm{M}$, et al. Epidemiology, prevention and control strategies of coronavirus COVID 19 in Iran: A systematic review. Open Access Maced J Med Sci. 2020;8(1):480-8. https://doi.org/10.3889/oamjms.2020.5479

24. Sikkema RS, Pas SD, Nieuwenhuijse DF, Toole ÁO, Verweij J, Van Der Linden A, et al. Articles COVID-19 in health-care workers in three hospitals in the south of the Netherlands: A cross-sectional study. Lancet Infect Dis. 2020;20(11):1273-80. PMid:32622380

25. Verbeek JH, Rajamaki B, ljaz S, Sauni R, Toomey E, Blackwood B, et al. Personal protective equipment for preventing highly infectious diseases due to exposure to contaminated body fluids in healthcare staff. Cochrane Database Syst
Rev. 2020;5:CD011621. https://doi.org/10.1002/14651858. cd011621.pub5

PMid:27093058

26. Maskari ZA, Blushi AA, Khamis F, Tai AA, Salmi IA, Harthi HA, et al. Characteristic of healthcare workers infected with COVID-19, a cross-sectional observational study. Int J Infect Dis. 2021;102:32-6. https://doi.org/10.1016/j.ijid.2020.10.009 PMid:33039607

27. Kim R, Nachman S, Fernandes R, Meyers K, Taylor M, LeBlanc D, et al. Comparison of COVID-19 infections among healthcare workers and non-healthcare workers. PLoS One. 2020;15(12):e0241956. https://doi.org/10.1371/journal. pone. 0241956

PMid:33296367

28. Yang JY, Parkins MD, Canakis A, Aroniadis OC, Yadav D, Dixon RE, et al. Outcomes of COVID-19 among hospitalized health care workers in North America. JAMA Netw Open. 2021;4(1):e2035699. https://doi.org/10.1001/ jamanetworkopen.2020.35699

PMid:33507259

29. World Health Organization. Coronavirus Disease (COVID-19) Pandemic. Available from: https://www.who.int/news-room/ commentaries/detail/criteria-for-releasing-covid-19-patientsfrom-isolation;2020. [Last accessed on 2020 Jul 13]. 


\section{Appendix}

\begin{tabular}{ll}
\hline HCW ID code: & Date of suspicion: \\
\hline Gender & Age \\
Male & \\
Female & \\
\hline
\end{tabular}

\section{Comorbidity:}

DM $\square$ HTN $\square$ Renal $\square$ Liver $\square$ Cardiac $\square$ Pulmonary disease or allergy $\square$ COPD $\square$ Smoking $\square$ Others $\square$ Job title:

Location of work: Frontline $\square$ Non frontline

Work experience: $<5$ years $\square>5$ years

Work hours: $<12 \mathrm{~h} \square>12 \mathrm{~h}$

Received IC training: Yes $\square$ No

Type of training: Diploma $\square$ Course $\square$ Lecture $\square$ Online training

Timing between exposure and appearance of symptoms (if known):

Contact with COVID-19 case: Yes $\square$ No

Place of Contact: In the hospital $\square$ Outside the hospital

Procedure done:

Talking with patient $\square$ Intubation/suction $\square$ Examination $\square$ Sampling $\square$ Others $\square$ Specify

Case presentation: Asymptomatic $\square$ Symptomatic

Symptom:

Fever $\square$ Sore throat $\square$ Cough $\square$ Dyspnea $\square$ Bone/muscle ache $\square$ Headache $\square$ Anosmia $\square$ Diarrhea Pneumonia $\square$ Others $\square$ specify

Result PCR test: Positive $\square$ Negative

Management of infected worker:

Hospitalization at ward Hospitalization at ICU Home isolation

Glossary:

Frontline HCWs:

Frontline HCWs are defined as a worker who deal directly by any means, with suspected or et confirmed COVID-19 cases through patient intake, screening, inspection, testing, transport, treatment, nursing, specimen collection, and examination.

\section{Overloaded HCWs:}

Classified by working hours; $12 \mathrm{~h}$ or more of continuous work is considered work overload.

\section{Hot Areas:}

These are crowded areas where direct contact with suspected COVID-19 patients for prolonged time is non- avoidable with frequent aerosol generating procedures such as; triage area, emergency room, operation rooms and intensive care unit (ICU). 